\title{
Cupping (Hijama) in Skin diseases with positive Koebner's Phenomenon: What is New?
}

\author{
Khalifa E. Sharquie ${ }^{1}$, Fatema A. Al-Jaralla ${ }^{2}$
}

${ }^{1}$ Department of Dermatology, College of Medicine, University of Baghdad. Iraqi and Arab Board for Dermatology \& Venereology, Baghdad Teaching Hospital, Medical City, Baghdad, Iraq

${ }^{2}$ Department of Dermatology, College of Medicine, University of Baghdad, Baghdad, Iraq

\section{Correspondence:}

Fatema A. Al-Jaralla

Address: Department of Dermatology, College of Medicine, University of Baghdad, Baghdad, Iraq

Email: fatema.hamad@yahoo.com
Received: 31.05.2019,

Accepted: 13.06.2019

https://doi.org/10.5799/jcei/5856

\begin{abstract}
Background: Cupping therapy is an ancient traditional and complementary medicine used in the treatment of a broad range of medical conditions. It is very popular in many countries, especially in China, Korea, and Japan. It is used in the Muslim world, as well as in North Africa, Eastern Europe and Latin America. Cupping involves applying a heated cup to generate a partial vacuum that mobilizes the blood flow and promotes effective healing. Till now, there is no certain scientific base for using cupping in treating any medical problem especially skin diseases. Many skin diseases have tendency to appear at the site of injury or damage to the skin, the so called koebners phenomena. Our observation is that hijama induces skin diseases at the site of cupping in patients with skin dis-eases with koebner phenomenon. Objective: to present cases with koebner phenomenon after cupping with primary lesions seen at the site of cupping. Patients and method: a total of 24 male patients previously diagnosed with skin diseases; 16 with psoriasis, 6 with lichen planus, one patient with dermatitis herpetiformis, and one with seborrheic dermatitis. Results: All patients had stable skin diseases prior to cupping but new lesions appeared at the site of cupping around two weeks after the procedure. Conclusion: cupping should not be encouraged for skin diseases with positive koebner phenomenon commonly psoriasis and lichen planus and possibly contraindicated as till now there is no scientific basis for using this procedure.
\end{abstract}

Keywords: hijama, skin diseases, psoriasis, lichen planus, koebner phenomenon

\section{INTRODUCTION}

Cupping therapy (CT) is an ancient medical art practiced and sustained by various human civilizations. According to Hasan et al, CT was practiced in Egypt more than 5500 years ago, and was symbolized in ancient hieroglyphics [1]. Assyrians were the first recorded Arab practitioners around 3500 B.C. Wet cupping is also known as Hijama (Arabic: "sucking") or medicinal bleeding, where blood is drawn by local suction from a small skin incision; is a technique that returns an ill state to its original state $[2,3]$. Dry CT used to be practiced more commonly in the Far East, while wet CT was preferred in the Middle East and Eastern Europe [4,5].

Wet cupping, unlike dry cupping, requires a skin incision. The skin incision can be made either before or after cupping treatment. The cup is meant to suck out any impurities in the body which may include blood, toxins, fragmented blood cells, and tissue fluid. A partial vacuum is created in cups placed on the skin either by means of heat or suction. Practitioners of cupping believe this maneuver invigorates blood flow and promotes healing. Cupping is commonly used on back, neck, shoulders, and buttocks [6,7].

In recent years, cupping therapy is often applied for low back pain, chronic nonspecific neck pain, arthritis fibromyalgia, immune system diseases and metabolic diseases, high blood pressure, ischemic and inflammatory myocardial conditions, herpes zoster, Behçet disease, secondary amenorrhea, depression and anxiety, and acne vulgaris [8]. It is reported that anemia, factitial panniculitis and herpes viral infection are the most frequent adverse events in the observational studies [9]. Cupping is related to post-inflammatory hyperpigmentation [10] and keloid [11]. 


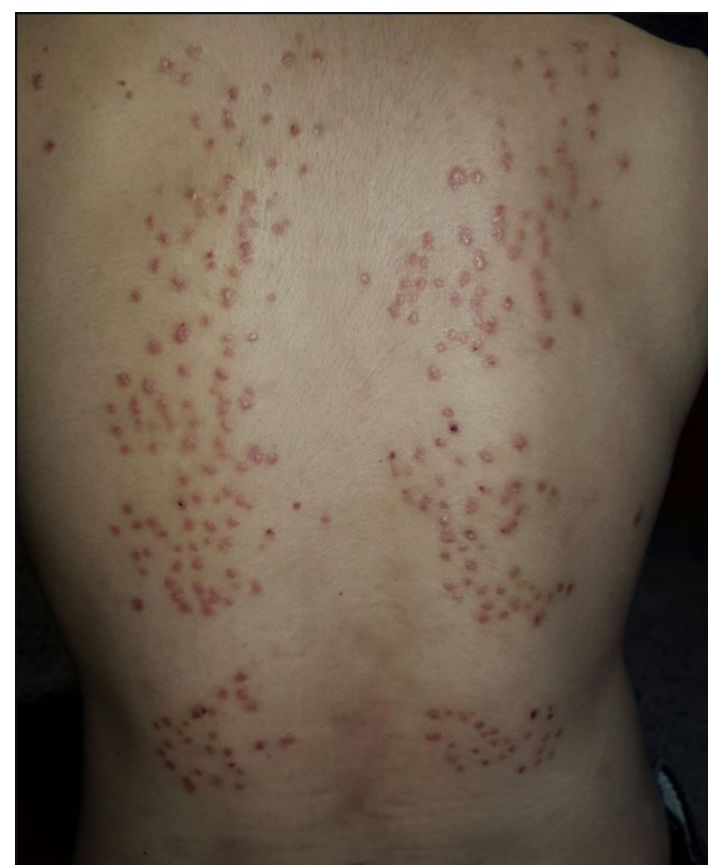

(a)

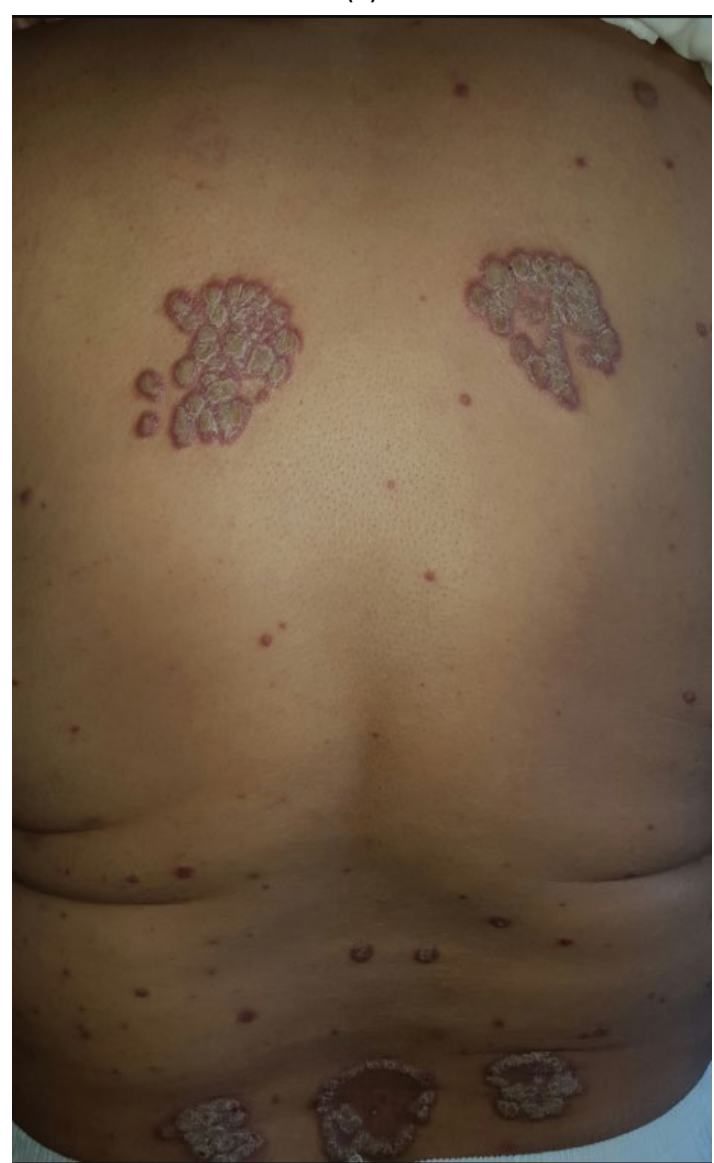

(b)

Figure 1. a. 27 year old man with psoriasis showing multiple well defined erythematous scaly papules and plaques arranged in circular pattern (koebnerization) at the sites of cupping b. 40 year old man also showing psoriatic plaques at the site of cupping.

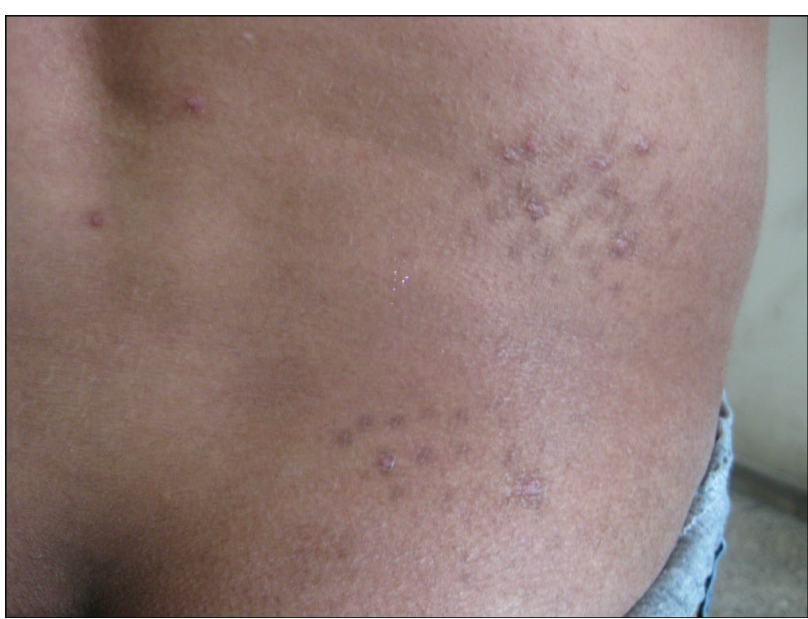

Figure 2. Koebner phenomenon with multiple well defined flat topped violaceous papules confined to the site of cupping in a patient with lichen planus, note the preexisting papules on the center of the lower back (black arrows).

Cupping therapy may be harmful for eczema [12]. There is a growing body of evidence suggests that the practice of Hijama carries a significant risk of blood borne infections, including HIV, hepatitis B, and hepatitis C infections [13]. Cupping of the cervical area may cause a hemorrhagic stroke by an acute rise in blood pressure [14]. These adverse events should raise attention to the safety of cupping therapy.

Many skin diseases have tendency to appear at the site of injury or damage to the skin, so called koebner phenomenon, such diseases like psoriasis, vitiligo, lichen planus, Darier disease, and bullous dermatoses arise at sites of cutaneous injury [15-18]. Today, the definition of koebner phenomenon has been extended even further to describe patients who did not have preexisting dermatosis before trauma or in whom the response did not reoccur on additional trauma.

Herein, we present 24 interesting cases with classic koebner phenomena after cupping with primary lesions seen at the site of cupping.

\section{PATIENTS AND METHOD}

A total of 24 male patients previously diagnosed with skin diseases were seen during the period 2009 and 2019; 16 patients with psoriasis, six with lichen planus, one patient with dermatitis herpitiformis, and one with seborrheic dermatitis. Their ages ranged between $25-40$ years with mean 32.5. All patients had stable skin diseases with prior to cupping but developed koebner phenomena around two weeks of cupping procedure. Patients had sought cupping for different reasons; mainly chronic back pain but also for dermatological causes attempting to cure their skin conditions. All patients did cupping on the back (Figures 1 and 2), except for three patients done on the lower extremities (Figures 3 and $\mathbf{4}$ ). 


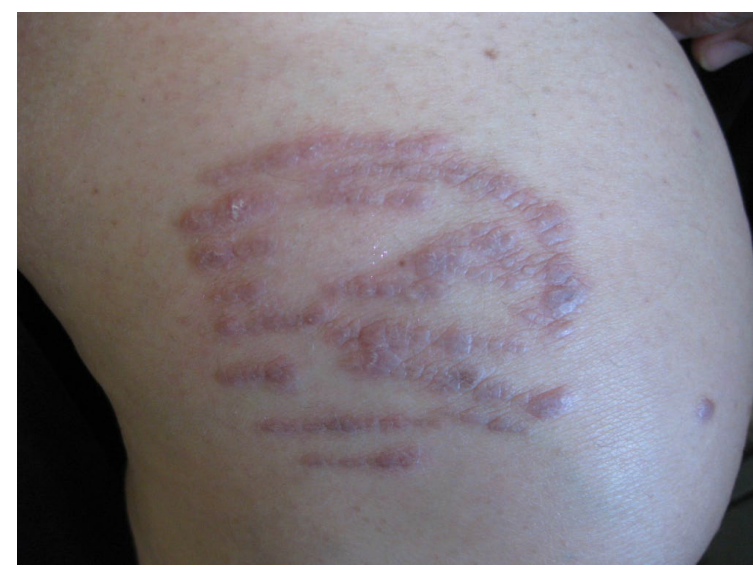

(a)

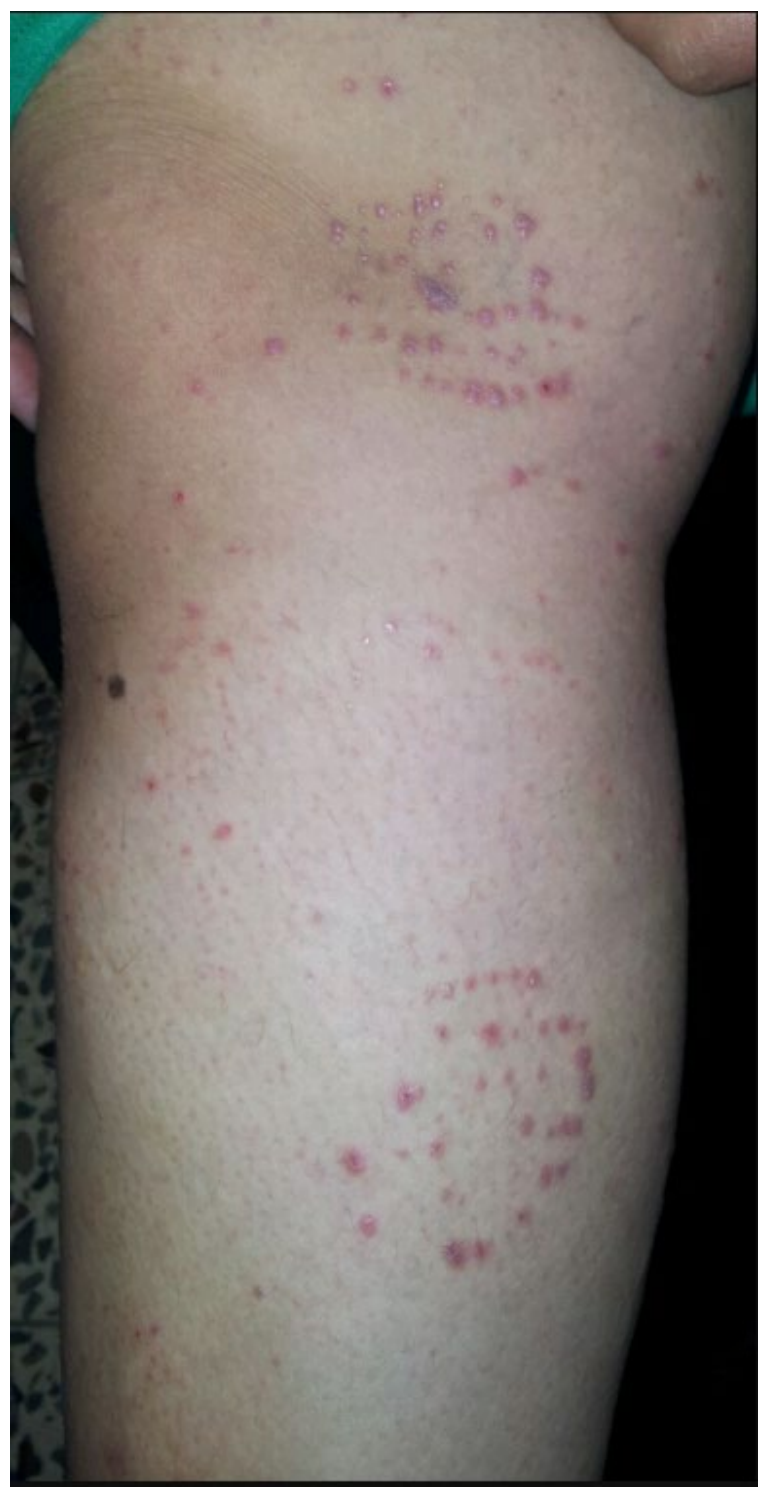

(b)

Figure 3. a. Medial aspect of the left lower thigh showing multiple well defined flat topped violaceous papules forming a plaque confined to the site of cupping in a patient with lichen planus. b. Another patient with lichen planus; showing obvious kobnerization at the medial aspect of the right lower extremity

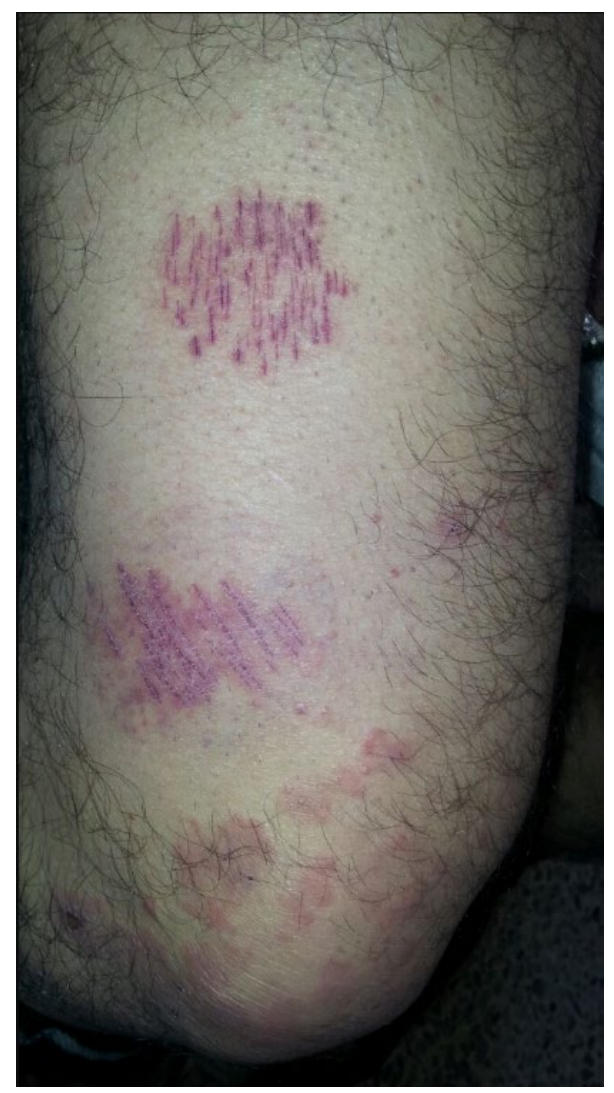

(a)

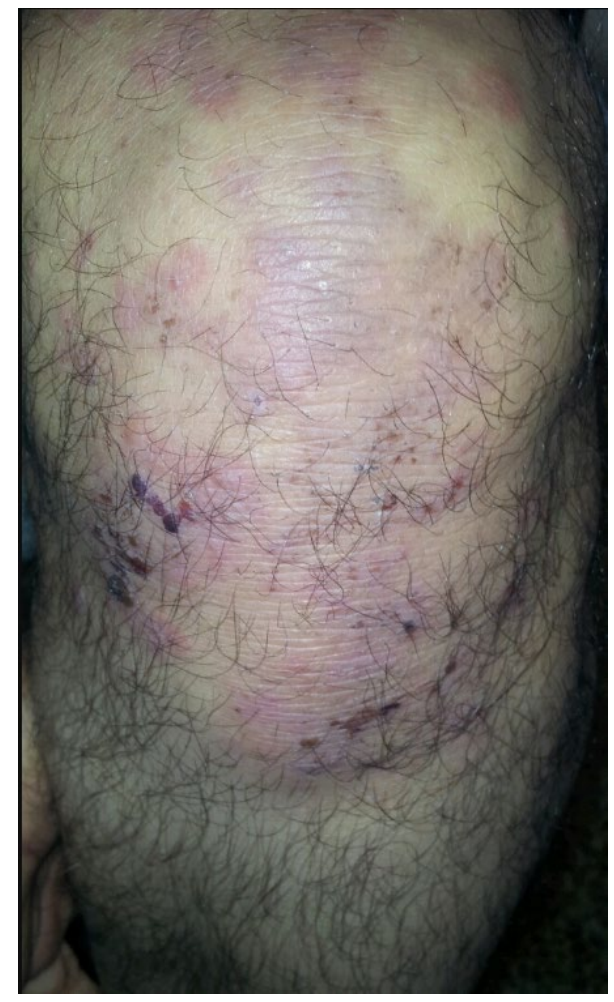

(b)

Figure 4. Patient with dermatitis herpitiformis developing koebner phenomena on the sites of cupping; $\mathbf{a}$ and $\mathbf{b}$ on the right thigh and right knee 


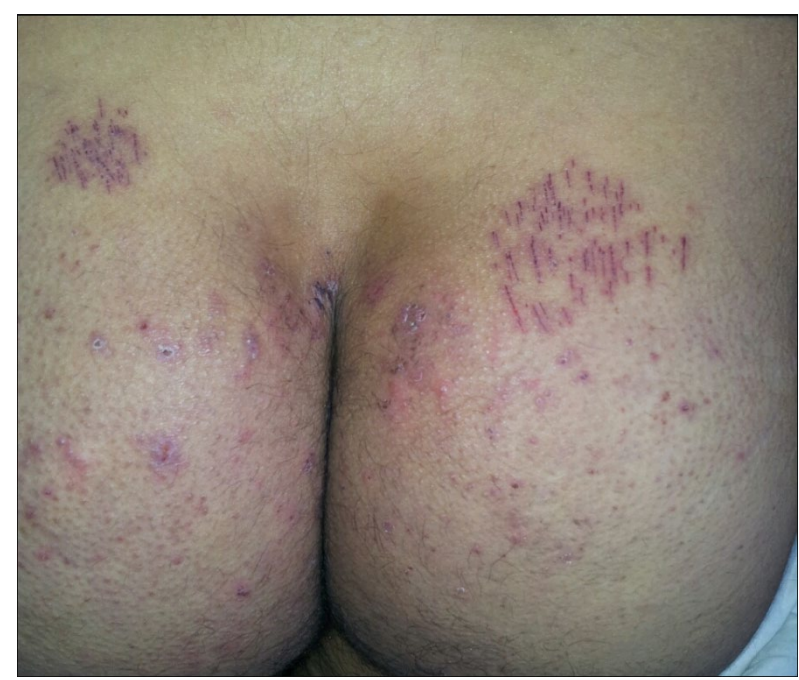

(c)

Figure 4 (continued). Patient with dermatitis herpitiformis developing koebner phenomena on the sites of cupping; $c$. on the lower back (note the preexisting excoriated vesicles grouped adjacent to the site of cupping

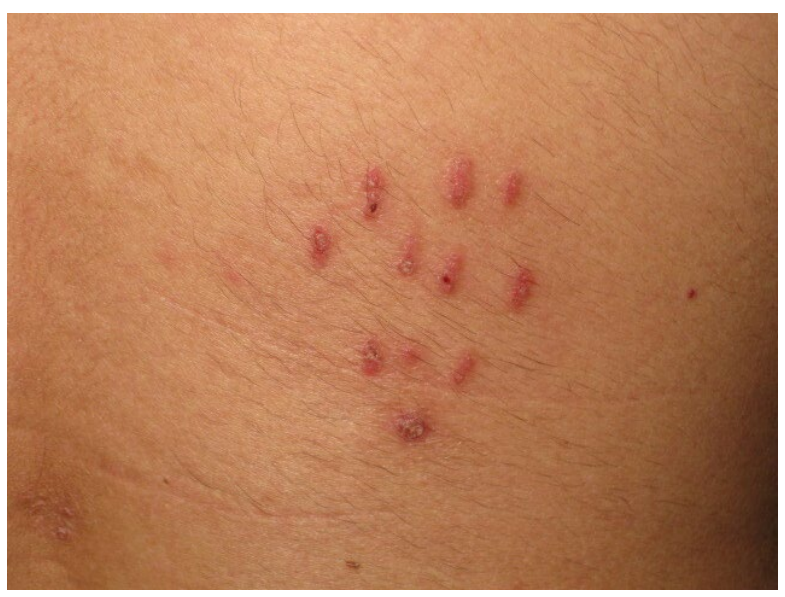

(a)

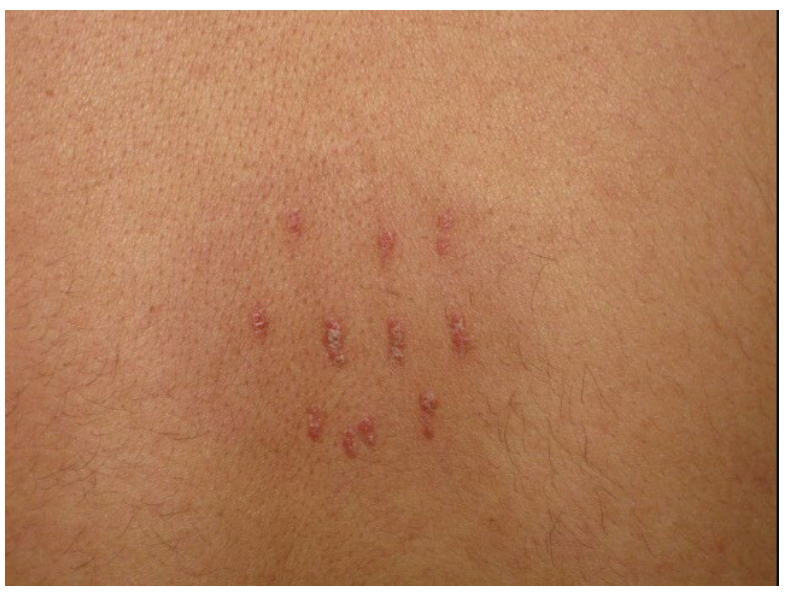

(b)

Figure 5. $\mathbf{a}$ and $\mathbf{b}$. patient with chronic stable psoriasis developed koebner phenomena on the back after cupping. Lesions assume the shape the small incisions done for bloodletting
A physical examination revealed circular plaques evenly spaced and of uniform size, most of the skin lesions took the shape of the small incisions done for bloodletting and all were similar to primary disease (Figure 5). On further inquiry, the patients admitted to undergo wet cupping procedure within the past few weeks.

\section{DISCUSSION}

Wet cupping has been practiced for centuries. Cupping therapy was hypothesized to adjust skin blood flow [19], make changes in biomechanical properties of the skin [20], increase immediate pressure pain thresholds in some areas [21], and adjusts serum P substance [22]. Cupping seems to play a role in the activation of complement system as well as modulation of cellular part of immune system [23]. However, further studies are needed to provide strong evidence that $\mathrm{CT}$ is effective in medical conditions associated with or without pain. Studies that estimate the costeffectiveness of CT in various diseases are also needed.

The Koebner phenomenon (KP) was first described in 1876 by Heinrich Koebner, a German dermatologist who was the founder of the dermatology department at the University of Breslau. He reported the formation of psoriasiform lesions in uninvolved skin of psoriatic patients after cutaneous trauma, such as excoriations, tattoos, and horse bites [24] the pathogenesis of Koebner phenomenon is unknown; however, there are immunologic, vascular, dermal, neural, genetic, hormonal, and infectious theories. Likewise, growth factors, drugs, cytokines, stress proteins, adhesion molecules, and autoantigens may also be involved $[25,26]$.

The efficacy of cupping therapy is controversial. ElDomyati and colleagues recognized cupping therapy as an effective alternative or complementary therapy for certain dermatoses but not for eight cases of psoriasis in an Egyptian study [27]. There has been one other report in China of Koebner phenomenon induced by cupping therapy in a psoriasis patient in the past literature [28]. But the present study does suggest that cupping increases severity of skin diseases with koebner phenomenon most commonly patients with psoriasis and lichen planus.

\section{CONCLUSION}

Patients with skin diseases may develop localized skin reaction at the site of trauma through koebnerization as a result of cupping therapy. Thus we do not recommend cupping therapy for patients with skin diseases with positive Koebner phenomenon but further studies are highly recommended to record all skin diseases where cupping is contraindicated.

Declaration of interest: The authors report no conflicts of interest.

Financial Disclosure: This study was an independent study and not funded by any drug companies. 


\section{REFERENCES}

1. Hasan I, Ahmad T, Ahmad S. Management of hypertension by wet cupping therapy (Al-Hijamah): a case study. Int J Pharmacol Toxicol. 2014;4(1):24-27

2. Sheikho MA. Cupping: a prophetical medicine appears in its new scientific perspective. North Charleston: Create Space Independent Publishing Platform. 2011:12-15.

3. Albinali, Hajar (June 2004). "Traditional Medicine among Gulf Arabs Part II - Blood Letting”. Heart Views. 5(2): 74-85

4. Hanan SA, Eman SE. Cupping therapy (Al-Hijama): it's impact on persistent non-specific lower back pain and client disability. Life Sci J. 2013;10(4s):631-642.

5. Stovner LJ, Hagen K, Jensen R, Katsarava Z, Lipton R, Scher A, Steiner T, Zwart JA. The global burden of headache: a documentation of headache prevalence and disability worldwide. Cephalalgia. 2007;27(3): 193-210.

6. Cui S, Cui J. Progress of researches on the mechanism of cupping therapy. Zhen Ci Yan Jiu. 2012;37:506-510.

7. Chen $\mathrm{C}, \mathrm{Yu} \mathrm{H}$. Acupuncture, electrostimulation, and reflex therapy in dermatology. Dermatologic Therapy. 2003;16(2):87-92.

8. Bamfarahnak $\mathrm{H}$, Azizi A, Noorafshan A, Mohagheghzadeh A. A tale of Persian cupping therapy: 1001 potential applications and avenues for research, Forsch Komplementmed. 2014;21:42-47.

9. Kim TH, Kim KH, Choi JY, Lee MS. Adverse events related to cupping therapy in studies conducted in Korea: a systematic review, Eur J Integr Med, 2013;6:434-40.

10. Lee SJ, Chung WS, Lee JD, Kim HS. A patient with cupping-related post-inflammatory hyperpigmentation successfully treated with a $1,927 \mathrm{~nm}$ thulium fiber fractional laser, J Cosmet Laser Ther, 2014;16:66-8.

11. Park TH. Keloid on scapular area secondary to therapeutic dry cupping, Int Wound J, 2013. (doi: 10.1111/iwj.12184).

12. Hon KLE, Luk DCK, Leong K, Alexander KCF. Cupping therapy may be harmful for eczema: a PubMed search, Case Rep Pediatr, 2013;2013. (doi: $10.1155 / 2013 / 605829)$.

13. R Abdul, B Noor Ul-Ain, A Muhammad. Practice of cupping (Hijama) and the risk of bloodborne infections, Am J Inf Cont, 2014;42:1139.
14. Blunt SB, Lee HP. Can traditional "cupping” treatment cause a stroke? Med Hypotheses, 2010;74:945-9.

15. Hameed A, Khan AA. Koebner phenomenon in pemphigus vulgaris. Br J Dermatol 1996;135:152-3.

16. Hebra F. Disease of the skin. New Sydenham Society 1874:180.

17. Preissmann M. The provocative action of light in Darier's disease. Ann Dermatol Syphilol 1946:188.

18. Rosenberg EW, Noah PW. The Koebner phenomenon and the microbial basis of psoriasis. J Am Acad Dermatol 1988;18:151-8

19. Liu W, Piao S, Meng X, Wei LH. Effects of cupping on blood flow under skin of back in healthy human, World J Acupuncture Moxibustion, 2013;23:50-2.

20. Norouzali T, Roostayi MM, Dehghan MF, Abbasi M, Akbarzadeh BA, Khaleghi MR. The effects of cupping therapy on biomechanical properties in wistarrat skin, J Res Rehab Sci, 2014;9:841-51.

21. Emerich M, Braeunig M, Clement HW, Lüdtked R, Hubera R. Mode of action of cupping-local metabolism and pain thresholds in neck pain patients and healthy subjects, Complement Ther Med, 2014;22:148-58.

22. Tian H, Tian YJ, Wang B, Yang L, Wang YY, Yang JS. Impacts of bleeding and cupping therapy on serum $\mathrm{P}$ substance in patients of postherpetic neuralgia, Zhongguo Zhen Jiu, 2013;33:678-81.

23. Khalil AM, Al-Qaoud KM, Shaqqour HM. Investigation of selected immunocytogenetic effects of wet cupping in healthy men, J Complement Med Drug Discov, 2013;3:51-7.

24. Köbner H. Zur Aetiologie der Psoriasis. Vjschr Dermatol 1876:559.

25. Camargo CM, Brotas AM, Ramos-e-Silva M, Carneiro S. Isomorphic phenomenon of Koebner: facts and controversies. Clin Dermatol 2013;31:741-9. (doi: 10.1016/j.clindermatol.2013.05.012).

26. Sagi L, Trau H. The Koebner phenomenon. Clin Dermatol 2011;29:231-6. (doi: 10.1016/j.clindermatol.2010.09.014).

27. El-Domyati M, Saleh F, Barakat M, Mohamed N. Evaluation of cupping therapy in some dermatoses. Egypt Dermatol Online J 2013;9:2.

28. Yu RX, Hui Y, Li CR. Köebner phenomenon induced by cupping therapy in a psoriasis patient. Dermatol Online J 2013;19:18575. 